TITLE:

\title{
Critical response evaluation of damped bilinear hysteretic SDOF model under long duration ground motion simulated by multi impulse motion
}

\section{$\operatorname{AUTHOR}(\mathrm{S})$ :}

Akehashi, Hiroki; Kojima, Kotaro; Farsangi, Ehsan Noroozinejad; Takewaki, Izuru

\section{CITATION:}

Akehashi, Hiroki ...[et al]. Critical response evaluation of damped bilinear hysteretic SDOF model under long duration ground motion simulated by multi impulse motion. International Journal of Earthquake and Impact Engineering 2018, 2(4): 298-321

\section{ISSUE DATE:}

2018-12

URL:

http://hdl.handle.net/2433/241739

\section{RIGHT:}

This is the accepted Manuscript of the article, which has been published in final form at

http://doi.org/10.1504/IJEIE.2018.099361.; The full-text file will be made open to the public on 1 December 2019 in

accordance with publisher's 'Terms and Conditions for Self-Archiving'; This is not the published version. Please cite only the published version,; この論文は出版社版でありません。引用の際には出版社版をご確認ご利用ください。 


\title{
Critical Response Evaluation of Damped Bilinear Hysteretic SDOF Model under Long Duration Ground Motion Simulated by Multi Impulse Motion
}

Hiroki Akehashi ${ }^{1}$, Kotaro Kojima ${ }^{2}$, Ehsan Noroozinejad Farsangi ${ }^{3}$, Izuru Takewaki ${ }^{1 *}$

${ }^{1}$ Department of Architecture and Architectural Engineering, Graduate School of Engineering, Kyoto University, Kyotodaigaku-Katsura, Nishikyo, Kyoto 615-8540, Japan

2 Faculty of Design and Architecture, Kyoto Institute of Technology, Matsugasaki, Sakyo, Kyoto 6068585, Japan

${ }^{3}$ Department of Earthquake Engineering, School of Civil Engineering, Graduate University of Advanced Technology (KGUT), Kerman, Iran

* Correspondence: Izuru Takewaki, Department of Architecture and Architectural Engineering, Graduate School of Engineering, Kyoto University, Kyotodaigaku-Katsura, Nishikyo, Kyoto 6158540, Japan; E-mail takewaki@archi.kyoto-u.ac.jp

\begin{abstract}
Multi impulse is used as a representative of a series of many-cycle harmonic waves which substantially simulate the long-duration earthquake event. An analytical formulation is developed for the elasticplastic response of a single-degree-of-freedom (SDOF) damped structure with bilinear hysteresis under the critical multi impulse condition. Following the procedure for elastic-perfectly plastic models, a novel procedure using an energy balance law is introduced. In this procedure, under the multi impulse condition, only the free-vibration exists, hence the energy balance law can be applied easily. An approximate but effective treatment of the energy dissipated by the viscous damping is a new perspective. It is shown that based on an analytical solution, the critical maximum plastic deformation and the corresponding critical impulse timing can be determined in the steady state condition depending on the input values. To investigate the reliability and accuracy of the proposed approach, a comparison is made with the response analysis outcome to the corresponding sine wave as a representative of the long-duration earthquake ground motion (GM).
\end{abstract}

Keywords: Seismic response, Critical excitation, Critical response, Bilinear hysteresis, Viscous damping, Long-duration GM, Resonance, Multi impulse. 


\section{Introduction}

Versatile documentation of recent GM recording enabled the classification of types of earthquake GMs (Abrahamson et al. 1998). One is a near-fault GM, the second is a random-type motion resulting from a subduction-zone fault and the third is determined as a long-duration (mostly far-fault) GM. Other factors which influence the classification are the soil types (soft and stiff soil, rock) at recording stations and fault mechanisms. Long-period GMs are new-type motions which have been under investigation in the recent studies (see Takewaki et al. 2011). Various influences of near-fault GMs on structural responses have also been discussed in litrature (for example Bertero et al. 1978, Kalkan and Kunnath 2006). Fling-step and forward-directivity are the main concepts for characterizing such near-fault GMs. Earthquake GMs provided by Northridge (USA, 1994), Hyogoken-Nanbu (Kobe, 1995), Chi-Chi (Taiwan, 1999), Bam (Iran, 2003) and Kumamoto (Japan, 2016) surprised many earthquake structural engineers and brought much interest.

The fling-step (fault-parallel) and forward-directivity (fault-normal) motions have been simulated in terms of two or three wavelets. Since the numerical formulation of elastic-plastic response is somehow complicated and many parameters have influence on that (e.g. period and amplitude of pulse, duration, ratio of pulse frequency to structure natural frequency, change of equivalent natural frequency for the increased input level), GM most of the previous investigations have considered a simplified elastic response only.

To tackle such complicated problem, an innovative approach using impulses as inputs, i.e. the double impulse, has been recently introduced by Kojima and Takewaki (2015a). It was assumed that the double impulse characterizes the fling-step GM, if the magnitude is tuned in an appropriate manner. In the next stage a closed-form formulation was proposed for calculating the maximum elastic-plastic response of a structure under the 'critical double impulse'. It was demonstrated that under the considered double impulse, only the free-vibration could happen and the energy balance theory at two key vibration states (maximum deformation state and maximum velocity state) helps to derive such analytical expression. It was also shown that according to impulses input level, after the $1^{\text {st }}$ or $2^{\text {nd }}$ impulses the maximum elastic-plastic deformation can be achieved. The accuracy of the proposed approach was investigated by comparing the proposed expressions with the results of time-history (TH) response analysis to the corresponding one-cycle sine wave as a representative of the fling-step earthquake motion. The magnitude of the double impulse was tuned so that the comparison is valid. In this tuning, the maximum Fourier amplitude of the double impulse was set so as to be equal to that of the corresponding one-cycle sine wave. In the study conducted by Kojima and Takewaki (2015b), the theory for the forward-directivity was derived by extending the proposed approach on fling-step expressions

As another approach, Casapulla et al. (2010) and Casapulla and Maione (2016) considered the multiple sequence of impulses for the rocking response of a rigid block and derived the resonant response. They introduced two types of multiple impulses, i.e. with gradually increasing intervals for resonance and with equal intervals. 
73 The closed-form or analytical expressions for the elastic-plastic responses to earthquake GMs have been derived in the past only for the steady-state responses or transient responses to a simple harmonic wave (Caughey 1960a, b, Roberts and Spanos 1990, Liu 2000). In general, a complexity will be imposed when there is a forced input in term of the harmonic wave, even for a simple solution in resonant and non-resonant cases. Since $20^{\text {th }}$ century, the seismic resistant design approach and developments considered the resonance phenomenon as a critical case in damage analysis of structures/infrastructures. In general, the resonant frequency should be analyzed for a specified input level by parametrically changing the input frequency in the response to a harmonic wave (Caughey 1960a, b, Iwan 1961, 1965a, b, Roberts and Spanos 1990, Liu 2000). It is desirable that no computational iteration is needed in the analysis stage. This can be achieved by introducing the multi impulse. When using the multi impulse, the free vibration analysis can be conducted without the specification of input frequency before the second impulse is applied. The analysis of resonant case can be done utilizing an energy balance law without the solution of differential equations. The timing of the impulses can as well be obtained as the time with zero restoring force. To calculate the maximum elastic-plastic response after impulse can be obtained by equating the initial kinetic energy to the combined elastic strain and hysteretic energies. This methodology can be used to determine the critical response only. The critical resonant frequency can be found automatically for the gradually increasing input level of the multi impulse.

In a recent investigation by (Kojima and Takewaki 2017), a closed-form critical response was derived for an undamped bilinear hysteretic SDOF model subjected to multi impulse. In another paper (Kojima et al. 2017), a closed-form critical response was obtained for a damped elastic perfectly-plastic SDOF model subjected to double impulse. However, the damping force-deformation relation was modeled by a quadratic function. The first novelty of the current work is the combination of damping and bilinear hysteresis for multi impulse which has been initially developed by authors in (Akehashi et al., 2018a). Furthermore, the modeling of the damping force-deformation relation by an elliptical function is another new point from the accuracy viewpoint.

Figure 1 shows a recorded resonant response in a tall building structure located in Osaka, Japan during the 2011 Tohoku earthquake. Although only non-structural elements were significantly damaged in this structure, no clear damage in structural components was observed. However, the viewpoint of 


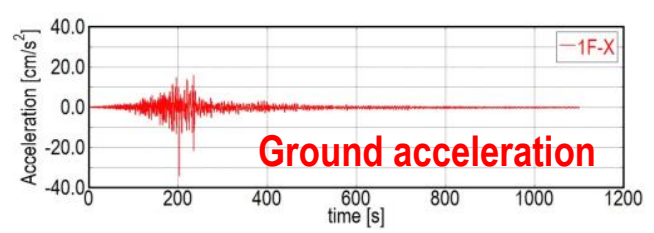

Long-duration, long-period ground motion of about 8 cycles is resonant with super high-rise building in Osaka
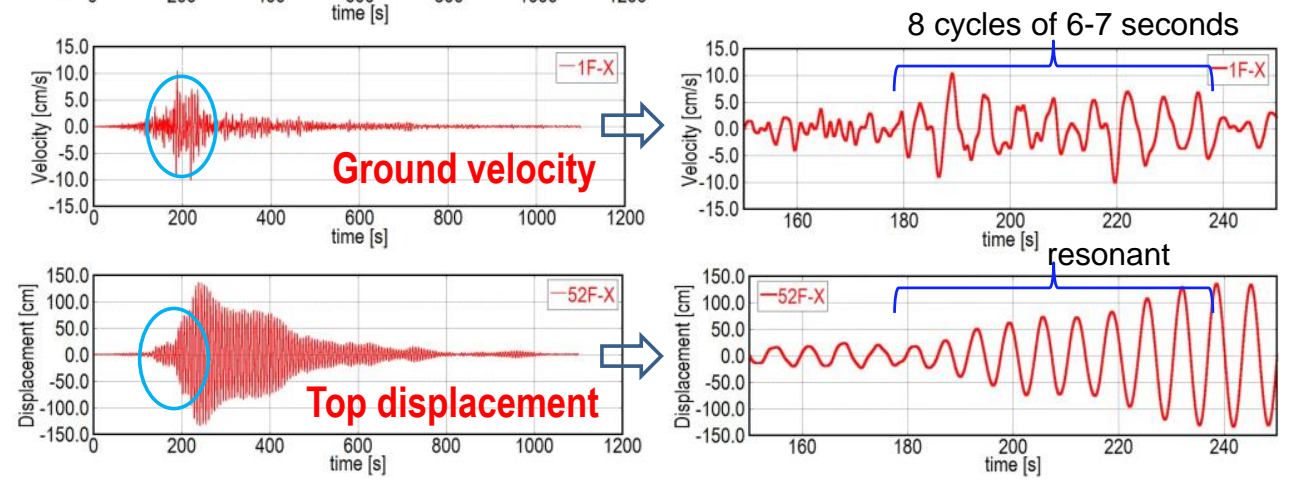

Figure 1 Recorded long-duration, long-period GM in a super tall building in Osaka, Japan during the 2011 off the Pacific coast of Tohoku earthquake and resonant response at top (Takewaki et al. 2011)

The multi impulse as shown in Figure 2(a) is used as a representative of the multi-cycle sinusoidal wave simulating the long-duration GM and a closed-form formulation is obtained for the steady-state response of a damped bilinear hysteretic SDOF structure, as shown in Figure 2(b). A prototype damped bilinear hysteretic SDOF model investigated in this paper and the explanation is given in section 2 . The closed-form expressions are derived in section 3 for the elastic-plastic steady-state responses under the critical multi impulse and the critical time intervals in three cases (see Figure 2(c)). CASE 0 is where the model remains elastic, CASE 1 indicates the case where each impulse is given at the point of zero restoring-force in the unloading process and the other case, CASE 2, presents the case where each impulse is given at the point of zero restoring-force in the loading process. The accuracy of the proposed formulation is investigated by comparison between the calculated maximum response and the results of TH response analysis of a SDOF damped bilinear hysteretic system under the multi impulse. In section 3.5, the accuracy of using the multi impulse in place of the long-duration GM is also verified through the comparison with the response under the corresponding multi-cycle sinusoidal wave. In section 4, the response under the multi impulse with the critical time interval obtained in previous section is investigated to see whether in converges to the steady state condition.. It is clear that, if the vibration state converges to the steady state, the time interval between each set of consecutive impulses also converges to a constant value. The validity of the critical time interval calculated in previous sections is then investigated by TH response analysis for a SDOF damped bilinear hysteretic model under multi impulse with various impulse time intervals. The applicability and accuracy of the closed-form steady-state response are investigated in the final stage and the outcomes are presented in sections $6 \& 7$ respectively. The conclusions are summarized in Section 8. 


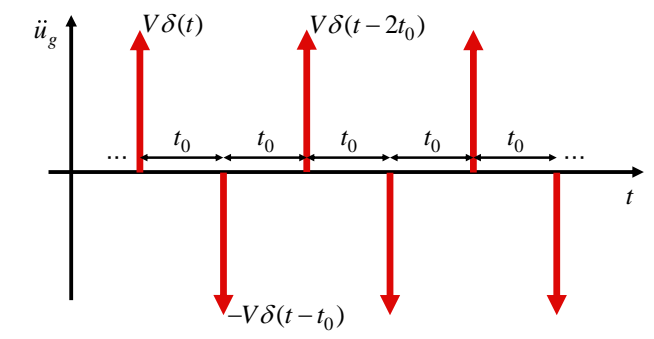

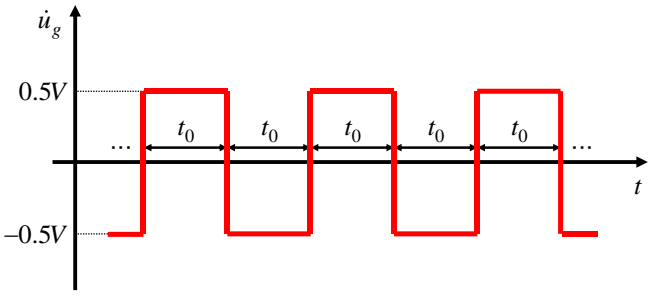

(a)

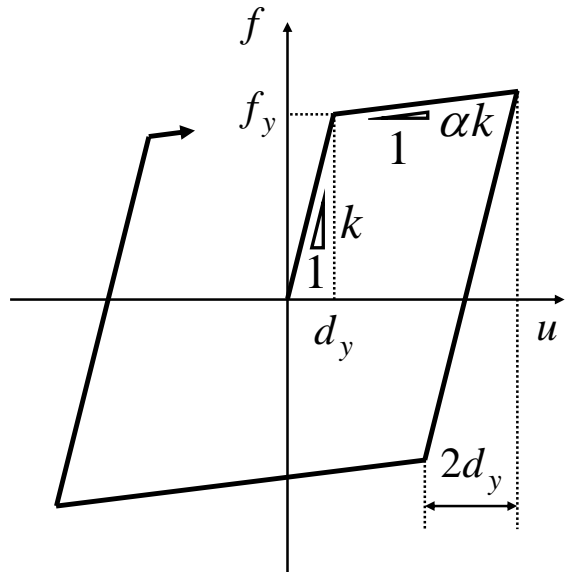

(b)

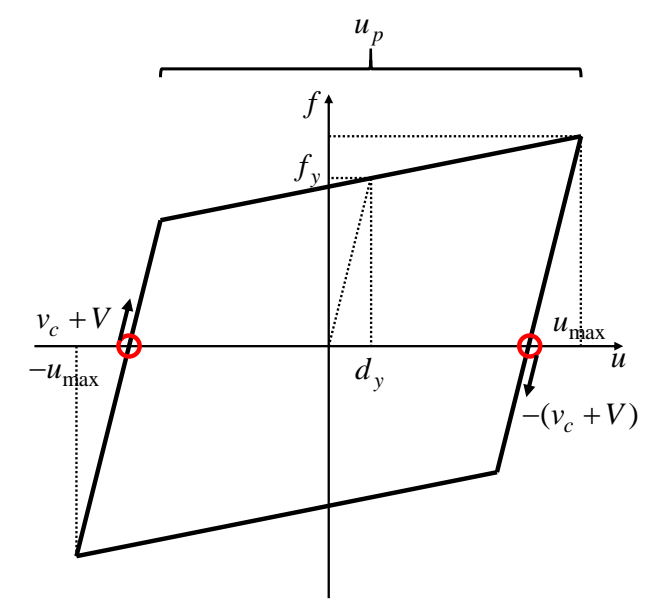

(c)

Figure 2 Multi impulse and bilinear hysteretic restoring-force characteristic: (a) Multi impulse with constant time interval $t_{0}$, (b) Bilinear hysteretic restoring-force characteristic, (c) Steady-state loop in restoring-force characteristic under critical multi impulse

\section{Damped bilinear hysteretic SDOF system}

A damped bilinear hysteretic SDOF system, as shown in Figure 2(b) should be considered. The model has a mass of $m$, stiffness of $k$ and damping coefficient of $c$ subjected to the multi impulse, as shown in Figure 2(a), with constant time interval. The given input velocity of each impulse is denoted by $V$ and the equal time interval between two neighboring impulses is quantified by $t_{0}$. Let $\alpha$ denote the ratio of the (post-yield to the initial elastic)- stiffness. In this paper, it is assumed that $\alpha>0$. The yield force and the yield deformation are characterized by $f_{y}$ and $d_{y}$ respectively. Let $\omega_{1}=\sqrt{\mathrm{k} / \mathrm{m}}$, $T_{1}=2 \pi / \omega_{1}, \omega_{1}^{\prime}=\sqrt{1-h^{2}} \omega_{1}, T_{1}^{\prime}=2 \pi / \omega_{1}^{\prime}, h, u$ and $f$, denote the undamped natural circular frequency, the undamped natural period, the damped natural circular frequency, the damped natural period, the damping ratio, the displacement of the mass relative to the ground (deformation of the system) and the restoring force of the model, respectively. The over-dot symbol used to describe time derivative. In section 3, these parameters will be treated as normalized ones. Let $V_{y}\left(=\omega_{1} d_{y}\right)$ as the input velocity of a single impulse at which the SDOF model at rest just attains the yield deformation after the first 
152

153

154

155

156

157

158

159

160

161

162

163

164

165

166

167

168

169

170

171

172

173

174

175

176

177

178

179

180

181

182

183

184

185

186

187

188

189

impulse. This parameter is related to the strength of the SDOF model. Numerical investigations are made in sections 4 through 7.

\section{Closed-form elastic-plastic steady-state response under critical multi impulse}

Kojima and Takewaki (2015a-c) derived analytical formulations for the critical elastic-plastic response of an undamped SDOF elastic-perfectly plastic model subjected to the several cases of double, triple and multi impulses. Furthermore, Akehashi et al. (2018a) obtained the analytical maximum response of a damped SDOF bilinear hysteretic model subjected to the double impulse. In this paper, the steadystate elastic-plastic response is derived through an analytical approach for a damped bilinear hysteretic SDOF model subjected to the critical multi impulse.

Each impulse causes the sudden change of velocity (the quantity $V$ ) of the mass and only free vibration arises after applying each impulse. Since the response of SDOF system subjected to multi impulse motion can be derived by a series of free vibrations, the model responses (plastic deformation amplitude and maximum deformation) can be obtained by using an energy balance law at two states without solving directly the equation of motion (EOM). It should be noted that at each impulse, the kinetic energy is transformed into a combination of strain and hysteretic energy.. The impulse critical timing corresponds to the point of zero restoring-force and only a kinetic energy exists at this state as mechanical energies. Considering this balance law, the model response in terms of maximum deformation can be obtained through a simplified approach. Kojima and Takewaki (2015c) derived an analytical expression to calculate plastic deformation amplitude and critical timing for the SDOF elastic-perfectly plastic model subjected to the critical multi impulse. The authors also developed a modified multi impulse motion, in which the second impulse is given at the point of zero restoringforce, to derive the analytical plastic deformation amplitude and critical timing. However, Kojima and Takewaki (2017) showed that the response of the undamped bilinear hysteretic SDOF model with a positive slope $\alpha>0$ is unstable under the initial impulses even under the condition that each impulse acts at the point of zero restoring-force; however, the response converges to a steady state as shown in Figure 2(c) after many repetitive impulses.

In this section, it is assumed that the system is in the steady state in which each impulse acts at the point of zero restoring-force and the analytical elastic-plastic response and critical timing can be derived by using the energy balance law. The response convergence is then investigated under multi impulse motion. It may be interesting to note that the convergence of the response under a harmonic wave into the steady state was also demonstrated by (Iwan 1961).

Plastic deformation level is the main criterion for classification of steady state under the critical multi impulse motion. Three cases have been defined and explained in the next sections.

\subsection{CASE 0: Elastic range}

First, consider CASE 0. Let us derive the plastic deformation amplitude and maximum deformation in the steady-state response of the damped bilinear hysteretic SDOF model using the energy balance 
190 law. Figure 3 shows the restoring force and damping force vs. deformation, to be used in the derivation

191

192

193

194

195

196

197

198

199

200

201

202

203

204

205

206

207

208

209

210

211

212

213

214

215

216

of the maximum steady-state response. The approximation of damping force-deformation realtion is defined based on quadratic function. It is shown that this approximation is in good conformity with the exact solution.

The velocity $v_{c}$ at the point of zero restoring-force is derived by solving the EOM in the unloading process. The velocity $v_{c}$ is expressed by

$$
v_{c} / V_{y}=E\left(u_{\max } / d_{y}\right)
$$

where,

$$
\begin{aligned}
& E=\exp \left[\left(-h / \sqrt{1-h^{2}}\right)(0.5 \pi+\phi)\right] \\
& \phi=\arctan \left(h / \sqrt{1-h^{2}}\right)
\end{aligned}
$$

The relation of damping force with deformation after the $2^{\text {nd }}$ impulse is evaluated using a quadratic function with the vertex $\left(u, f_{D}\right)=\left(u_{\max }, 0\right)$ and passing the point $\left(u, f_{D}\right)=\left(0, c\left(v_{c}+V\right)\right)$, as shown in Figure $3(\mathrm{~b})$. The $f_{D}$ (damping force) can be calculated as follows:

$$
f_{D}=c\left(v_{c}+V\right) \sqrt{1-\left(u / u_{\max }\right)} \quad\left(0 \leq u \leq u_{\max }\right)
$$

Integrating Eq. (4) from $u=0$ to $u=u_{\max }$, the work conducted by $f_{D}$ after impulse can be determined by Eq. (5) as follows:

$$
\int_{0}^{u_{\max }} f_{D} d u=\int_{0}^{u_{\max }} c\left(v_{c}+V\right) \sqrt{1-\left(u / u_{\max }\right)} d u=(2 / 3) c\left(v_{c}+V\right) u_{\max } .
$$

From Eq. (5), the energy balance law at the points of zero restoring-force and the the maximum deformation $u_{\max }$ leads to

$$
m\left(v_{c}+V\right)^{2} / 2=\left(k u_{\max }^{2} / 2\right)+(2 / 3) c\left(v_{c}+V\right) u_{\max } .
$$

By using Eqs. (1), (6), $u_{\max }$ can be obtained as

$$
u_{\max } / d_{y}=\left(-B_{0}+\sqrt{B_{0}^{2}-4 A_{0} C_{0}}\right) / 2 A_{0}
$$

where,

$$
\begin{aligned}
& A_{0}=1-E^{2}+(8 h / 3) E \\
& B_{0}=\{(8 h / 3)-2 E\}\left(V / V_{y}\right) \\
& C_{0}=-\left(V / V_{y}\right)^{2}
\end{aligned}
$$




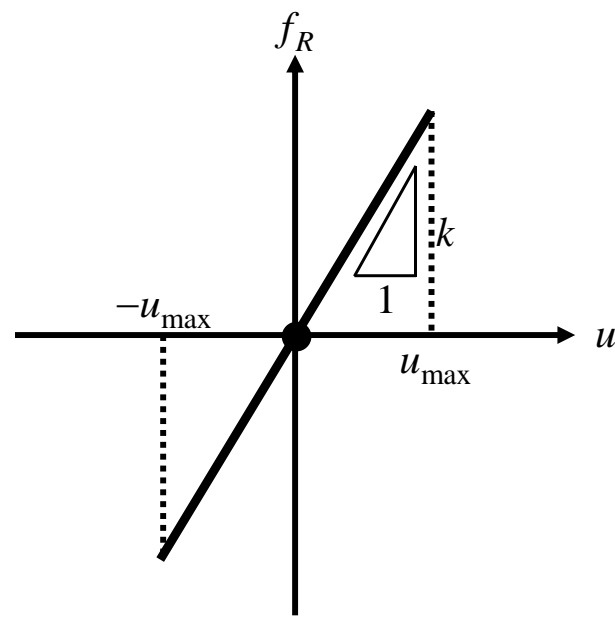

(a)

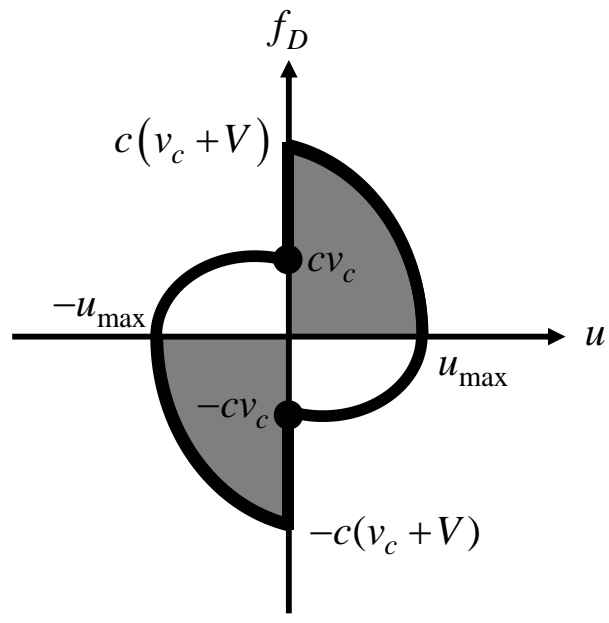

(b)

Figure 3 Restoring and damping forces vs. deformation(CASE 0) $\left[V / V_{y} \leq(4 h / 3)+\sqrt{\left(16 h^{2} / 9\right)+1}-E\right]$ : (a) Restoring force-deformation relation, (b) Quadratic function approximation of damping force-deformation relation.

Consider the boundary between CASE 0 and CASE 1 . This boundary input velocity level between this two cases can be provided by Eqs. (1), (6) and $u_{\max }=d_{y}$,

$$
V / V_{y}=(4 h / 3)+\sqrt{\left(16 h^{2} / 9\right)+1}-E
$$

\subsection{CASE 1: Impulse in unloading process}

Consider the next case, CASE 1. Let us derive the plastic deformation amplitude and maximum deformation in the steady-state response of the elastic-plastic SDOF model subjected to the critical multi impulse. Figure 4 shows the restoring force and damping force for deformation which are used in the derivation of the critical response in CASE 1. In this derivation, an energy approach is used again. A similar Quadratic function approximation used in CASE 0 is introduced to evaluate the work by the damping force.

The velocity $v_{c}$ at the zero restoring-force point in the unloading process can be formulated by finding a solution for the EOM in the unloading process. The velocity $v_{c}$ is expressed in terms of $u_{p}$ by

$$
v_{c} / V_{y}=E\left\{1+(\alpha / 2)\left(u_{p} / d_{y}\right)\right\}
$$

The relation of deformation and the damping force is approximated using a quadratic function with the vertex $\left(u, f_{D}\right)=\left(u_{\max }, 0\right)$ and passing the point $\left(u, f_{D}\right)=\left(-\{(1-\alpha) / 2\} u_{p}, c\left(v_{c}+V\right)\right)$, as shown in Figure 3(b). $f_{D}$ can then be obtained as follows:

$$
f_{D}=c\left(v_{c}+V\right) \sqrt{\left(u_{\max }-u\right) /\left(u_{\max }+\{(1-\alpha) / 2\} u_{p}\right)} \quad\left(-\{(1-\alpha) / 2\} u_{p} \leq u \leq u_{\max }\right)
$$

By integrating Eq. (13) from $u=-\{(1-\alpha) / 2\} u_{p}$ to $u=u_{\max }$, the work done by the damping force can be determined:

$$
\int_{-\{(1-\alpha) / 2\} u_{p}}^{u_{\max }} f_{D} d u=(2 / 3) c\left(v_{c}+V\right)\left\{(1-(\alpha / 2)) u_{p}+d_{y}\right\},
$$


244

245

246

247

248

249

250

251

252

253

254

255

256

257

258

259

260

261

262

263

264

265

266

267

268

269

270

271

where $u_{\max }=\left(u_{p} / 2\right)+d_{y}$. By using Eq. (14), the energy balance law at the zero restoring-force point and the point attaining the maximum deformation $u_{\max }$ (Figure 4: points A and C respectively) leads to

$$
m\left(v_{c}+V\right)^{2} / 2=(2 / 3) c\left(v_{c}+V\right)\left\{(1-(\alpha / 2)) u_{p}+d_{y}\right\}+(\operatorname{area} \text { of ABCD })
$$

The last term in Eq. 15 indicates the area of the quadrilateral in Figure 4(a). $u_{p}$ can be obtained through Eq. (16) by substituting Eq. (12) into Eq. (15),

$$
u_{p} / d_{y}=\left\{-B_{1}+\sqrt{B_{1}^{2}-4 A_{1} C_{1}}\right\} / 2 A_{1}
$$

where

$$
\begin{aligned}
& A_{1}=\left(\alpha^{2} / 4\right)\left(1-E^{2}\right)+(2 h / 3) E \alpha(2-\alpha) \\
& B_{1}=\left\{E+\left(V / V_{y}\right)\right\}\{(8 h / 3)-\alpha E\}-\alpha\left\{1+(4 h / 3)\left(V / V_{y}\right)\right\}+2 \\
& C_{1}=-\left(E+\left(V / V_{y}\right)\right)^{2}+(8 h / 3)\left(E+\left(V / V_{y}\right)\right)+1
\end{aligned}
$$

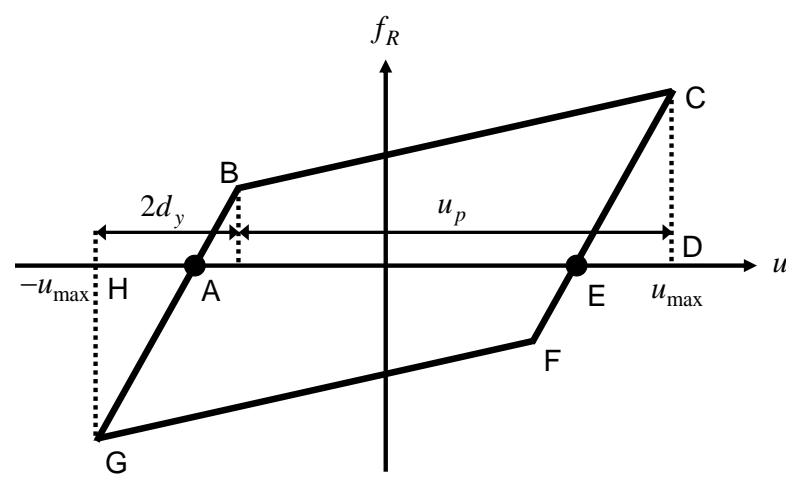

(a)

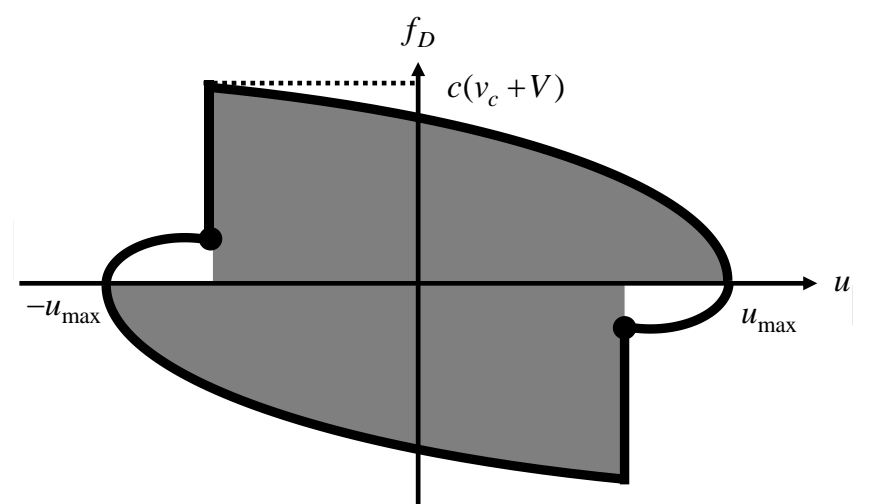

(b)

Figure 4 Restoring and damping forces for deformation in CASE 1 $\left[(4 h / 3)+\sqrt{\left(16 h^{2} / 9\right)+1}-E \leq V / V_{y} \leq\left\{(8 h / 3)+\sqrt{\left(64 h^{2} / 9\right)+4 \alpha}\right\} / \alpha-2 E\right]$ : (a) Restoring force vs.deformation relation, (b) Quadratic approximation of damping force-deformation relation.

On the boundary between CASE 1 and CASE 2, the zero restoring-force point is equal to the point of the yield initiation and each impulse acts at this point. The input velocity level on this boundary can be determined from Eqs. (12), (15) and $u_{p} / d_{y}=2 / \alpha$,

$$
V / V_{y}=\left\{(8 h / 3)+\sqrt{\left(64 h^{2} / 9\right)+4 \alpha}\right\} / \alpha-2 E
$$

\subsection{CASE 2: Impulse in loading process (second stiffness range)}

In the final stage, we may derive the steady-state response of the elastic-plastic hysteretic SDOF model subjected to the critical multi impulse motion by means of the energy balance law. Figure 5 shows the restoring force and damping force for deformation which are used in the formulation of the critical response in CASE 1 using an energy-based approach. The velocity $v_{m}$, i.e. the local maximum value 
272

273

274

275

276

277

278

279

280

281

282

283

284

285

286

287

288

289

290

291

292

293

294

295

296

297

298

of the velocity between the starting point of unloading and the zero restoring-force point, is not equal to the velocity $v_{c}$ at the zero restoring-force point, if $h>0$. In CASE 2 , the velocity $v_{m}$ can be derived by using an elliptical approximation of the damping force-deformation relation.

The velocity $v_{m}$ can as well be determined based on the energy approach. The damping forcedeformation relation between the starting point of unloading and the zero restoring-force point can be approximated by an ellipse which has the vertexes $\left(u, f_{D}\right)=\left(-u_{\max }, 0\right),\left(-(1 / \alpha-1) d_{y}-\left(h_{2} / \omega_{2}\right) v_{m}, c v_{m}\right)$, where $h_{2}=h / \sqrt{\alpha}, \omega_{2}=\sqrt{\alpha} \omega$. The work done by the damping force can be expressed by the quarter of the area of the ellipse. Therefore, the energy balance law between point $\mathrm{A}$ and $\mathrm{C}$ in Figure 5 leads to

$$
m v_{m}^{2} / 2+\pi c v_{m}\left\{\left(u_{p} / 2\right)+(2-(1 / \alpha)) d_{y}-\left(h_{2} / \omega_{2}\right) v_{m}\right\} / 4=(\text { area of ABCEFG })
$$

From Eq. (21), $v_{m}$ can be expressed in terms of $u_{p}$ by

$$
\frac{v_{m}}{V_{y}}=-\frac{\pi h}{4 Y}\left(\frac{u_{p}}{d_{y}}+4-\frac{2}{\alpha}\right)+\frac{1}{Y} \sqrt{\frac{\pi^{2} h^{2}}{16}\left(\frac{u_{p}}{d_{y}}+4-\frac{2}{\alpha}\right)^{2}-Y\left\{-\frac{\alpha}{4}\left(\frac{u_{p}}{d_{y}}\right)^{2}+(1-2 \alpha)\left(\frac{u_{p}}{d_{y}}\right)-\frac{1}{\alpha}\right\}}
$$

where

$$
Y=1+(1-\pi) h_{2}^{2}
$$

Assuming that the velocity between the starting point of unloading and the zero restoring-force point attains the local maximum value $v_{m}$ in the re-loading process, the term $v_{c}$ can be expressed in terms of $v_{m}$ by using the EOM.

$$
v_{c}=Z v_{m}
$$

where

$$
\begin{aligned}
& Z=\sqrt{1-h_{2}^{2}} \exp \left\{\left(-h_{2} / \sqrt{1-h_{2}^{2}}\right) \phi_{2}\right\} \\
& \phi_{2}=\arctan \left(h_{2} / \sqrt{1-h_{2}^{2}}\right)
\end{aligned}
$$

By solving the EOM between the zero restoring-force point and the point attaining the maximum deformation $u_{\max }, u_{p}$ is related to $v_{c}$ by

$$
\left(u_{p} / 2\right)+\left(d_{y} / \alpha\right)=\left(v_{c}+V\right) E_{2} / \omega_{2},
$$

where

$$
E_{2}=\exp \left\{\left(-h_{2} / \sqrt{1-h_{2}^{2}}\right)\left(\pi / 2-\phi_{2}\right)\right\}
$$

From Eqs. (22), (24) and (27), $u_{p}$ can be obtained as

$$
u_{p} / d_{y}=\left(-B_{2}+\sqrt{B_{2}^{2}-4 A_{2} C_{2}}\right) / 2 A_{2}
$$


299

300

301

302

303

304

305

306

where

$$
A_{2}=\frac{1-\left(E_{2}^{2} Z^{2} / Y\right)}{4}+\frac{\pi h E_{2}}{4 \sqrt{\alpha}} \frac{Z}{Y}
$$

$B_{2}=\frac{\pi h E_{2}}{\sqrt{\alpha}} \frac{Z}{Y}-\left(\frac{E_{2}}{\sqrt{\alpha}}+\frac{\pi h E_{2}^{2}}{2 \alpha} \frac{Z}{Y}\right)\left(\frac{V}{V_{y}}\right)+\frac{1+(1-2 \alpha)\left(E_{2}{ }^{2} Z^{2} / Y\right)}{\alpha}$

$$
C_{2}=\left(\frac{E_{2}}{\sqrt{\alpha}} \frac{V}{V_{y}}\right)^{2}-\left\{\frac{2 E_{2}}{\alpha \sqrt{\alpha}}+\frac{\pi h E_{2}^{2}}{2 \alpha} \frac{Z}{Y}\left(4-\frac{2}{\alpha}\right)\right\}\left(\frac{V}{V_{y}}\right)+\frac{\pi h E_{2}}{2 \alpha \sqrt{\alpha}} \frac{Z}{Y}\left(4-\frac{2}{\alpha}\right)+\frac{1-\left(E_{2}^{2} Z^{2} / Y\right)}{\alpha^{2}}
$$

The phenomenon of response divergence can occur where $V / V_{y} \geq(-2 \alpha+2) / \sqrt{\alpha}$ if $h=0$ (Kojima and Takewaki 2017). If $h>0$, the phenomenon cannot occur even for the large input velocity level $V / V_{y}$ (this will be explained in Section 3.5). A similar phenomenon exists under a sine wave input (Iwan 1961).

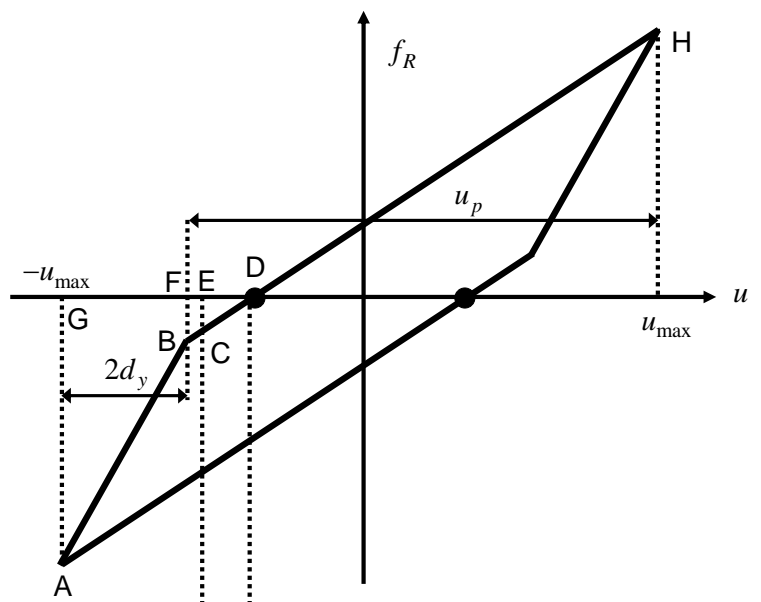

\subsection{Critical impulse timing}

Figure 5 Restoring force and damping force for deformation in CASE 2

In this section, the critical time interval $t_{0}{ }^{c}$ between two neighboring is derived. In contrast with the previous publications for the SDOF model without viscous damping, it may be complicated to 
314

315

316

317

318

319

320

321

322

323

324

325

326

formulate an analytical expression on the critical time interval between the impulses in CASE 1 and CASE 2. For this purpose, the TH response analysis is used for the critical multi impulse (each impulse acts at the zero restoring-force point) and the time interval is evaluated as the time up to the zero restoring-force timing. It seems compulsory to continue conducting the analysis until the response converges to a steady state.

Figure 6 depicts the normalized time interval $t_{0}{ }^{c}$ considering several levels of the input velocity, various post-yield stiffness ratios $\alpha=0.1,0.3,0.5$ and various damping ratios $h=0.05,0.1,0.2$. In CASE 0, the critical time interval is obtained as $t_{0}{ }^{c}$. In CASE 1 and CASE 2, as the damping ratio becomes larger, the plastic deformation $u_{p}$ and the critical time interval $t_{0}^{c}$ reduce. Finally, $t_{0}{ }^{c}$ converges to $\pi /\left(\omega_{2} \sqrt{1-h_{2}{ }^{2}}\right)$, a half of the damped natural period, because the second stiffness range greatly surpasses the initial elastic stiffness range. Therefore, the larger damping ratio leads to the longer, critical time interval. The sudden change in slope indicates the transition zone for CASEs 0,1 $\& 2$.

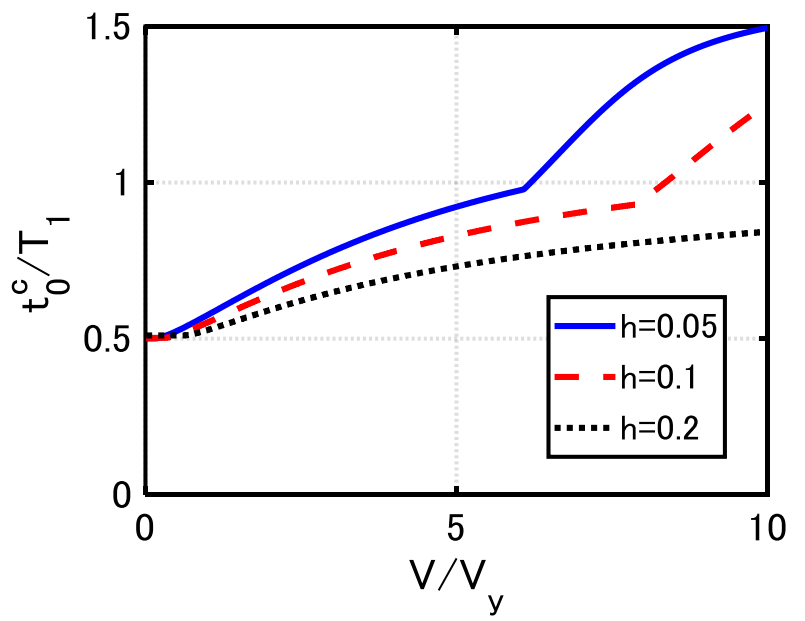

(a)

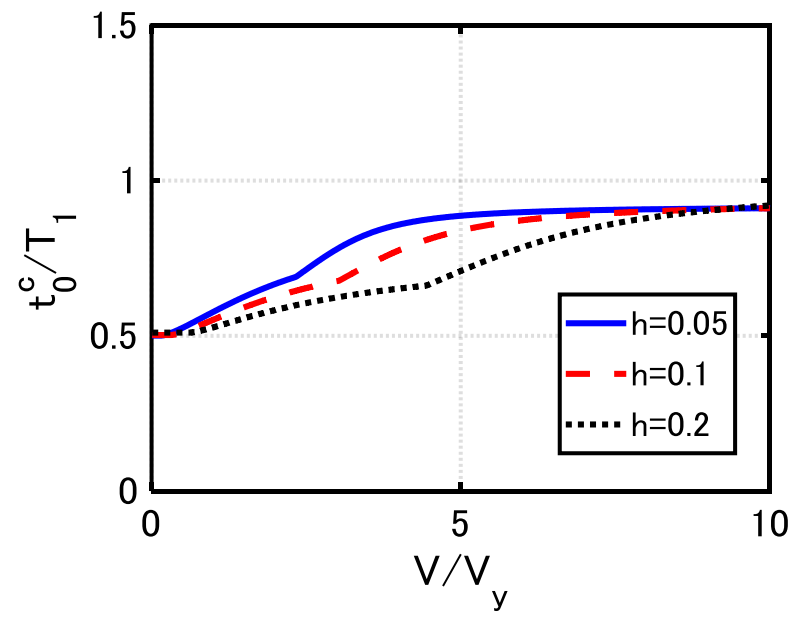

(b)

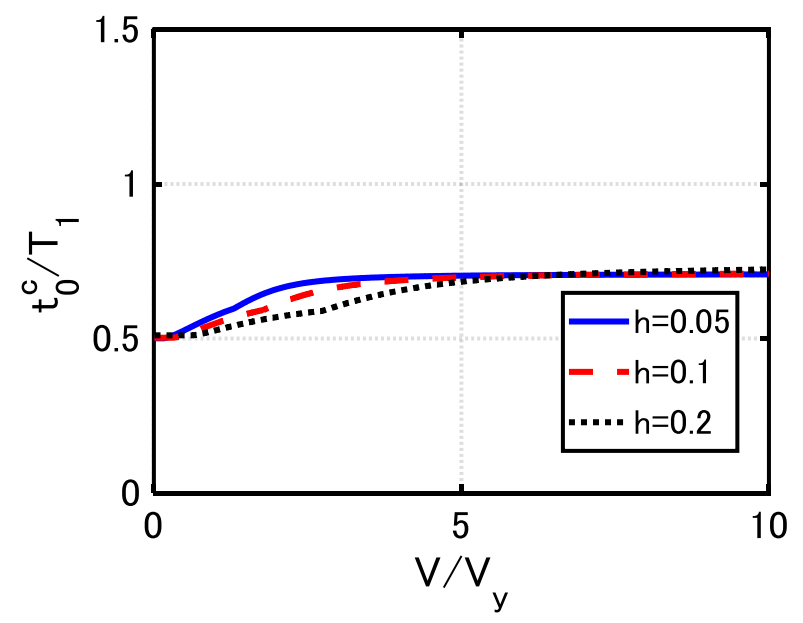

(c)

Figure 6 Critical impulse timing $t_{0}^{c} / T_{1}$ considering range of input level $V / V_{y}$ for various post-yield stiffness ratios $\alpha$, (a) $\alpha=0.1$, (b) $\alpha=0.3$, (c) $\alpha=0.5$. 
333

\subsection{Accuracy check of the proposed expression under multi impulse}

To check the accuracy of the proposed formulation under the multi impulse (since the damping forcedeformation relation is approximated, the expression is approximate), let us use the TH response analyses of the SDOF model subjected to the multi impulse and the amplitude-tuned multi-cycle sine wave.

In the evaluation process, it is substantial to tune the input levels between the multi impulse and the corresponding multi-cycle sine wave considering the equal maximum Fourier amplitude (Kojima and Takewaki 2017). The natural period $\left(T_{l}\right)$, the circular frequency $\left(\omega_{l}=2 \pi / T_{l}\right)$, the acceleration amplitude $\left(A_{l}\right)$ and the velocity amplitude $\left(V_{l}=A_{l} / \omega_{l}\right)$ of the corresponding sine wave are used in this section considering $T_{l}=2 t_{0}{ }^{c}$. It should be noted that the cycles number of the multi-cycle sine wave is half of the number of impulses. It is assumed that the steady state is existed only after a sufficient number (for example over 20 impulses) of impulses in the derivation of the response under the multi impulse motion. The input velocity level of the multi impulse can be expressed based on the acceleration amplitude of the corresponding multi-cycle sine wave as follows:

$$
V_{l}=A_{l} / \omega_{l}=(2 / \pi) V
$$

Figures 7-9 compare the maximum deformations of the SDOF models for $\alpha=0.1,0.3,0.5$ and $h=0.05,0.1,0.2$. The comparison indicates that TH response analysis under the critical multi impulse and the amplitude-tuned multi-cycle sine wave will approximate the damping force-deformation relation of the dashpot quite accurately. The response of the undamped SDOF with the small post-yield stiffness under the multi impulse and that under the sine wave do not correspond well with that for the large input level (Kojima and Takewaki 2017). The responses of the damped bilinear hysteretic SDOF model subjected to those inputs correspond better compared to the undamped model.

It is known that the displacement response of a linear elastic SDOF model to steady-state harmonic excitation is almost inversely proportional to the damping ratio $h$. From Figures 8 and 9 , it can be observed that $u_{\max }$ is almost inversely proportional to the damping ratio $h$ in the range of $V / V_{y} \geq 8$. With the large input level, the second stiffness range in the response greatly surpasses the initial elastic stiffness range and the model behaves like a linear elastic model of stiffness $\alpha k$. 


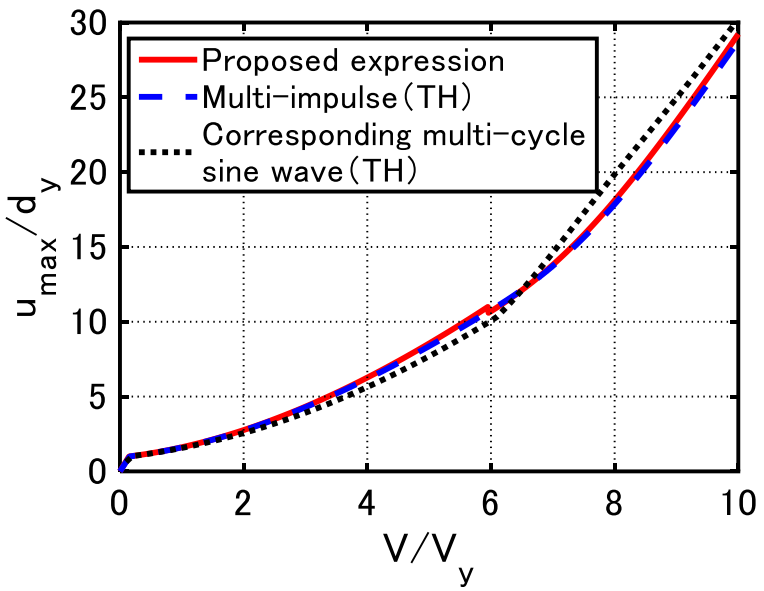

(a)

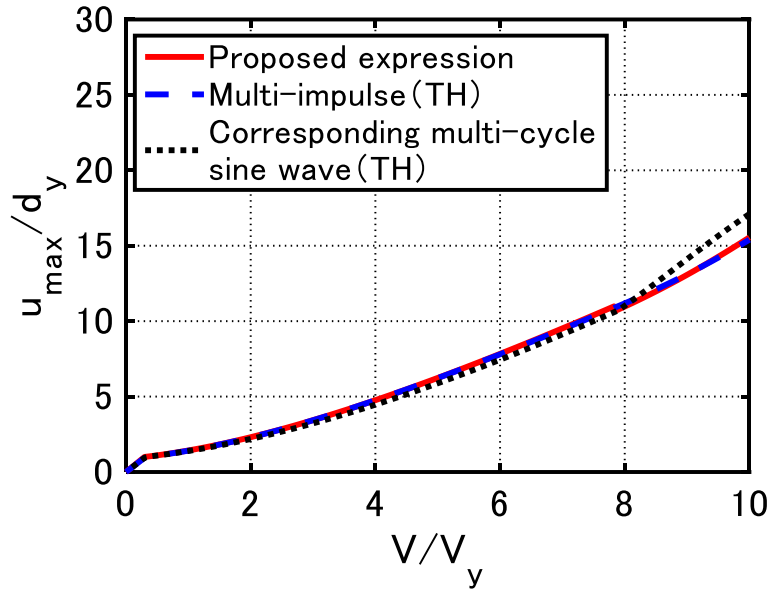

(b)

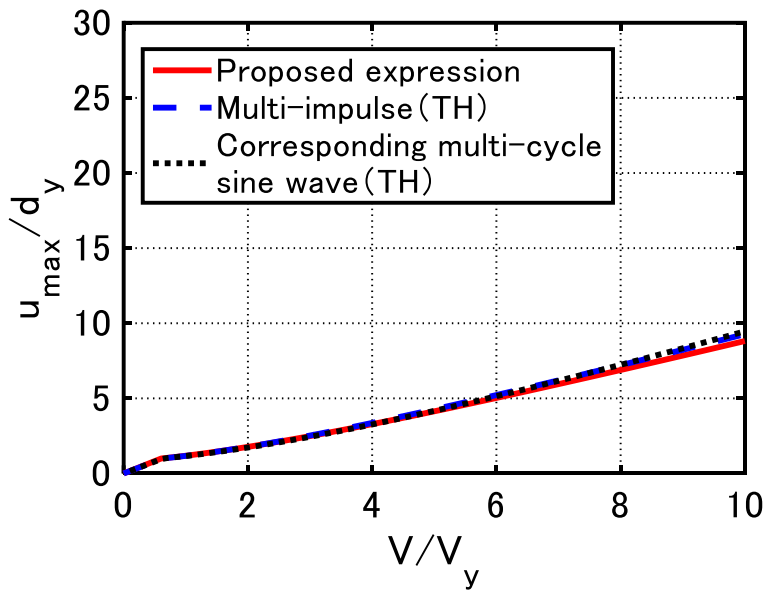

(c)

Figure 7 Comparison of maximum deformations for a sample model ( $\alpha=0.1$ ), TH response analysis under multi impulse and corresponding multi-cycle sine wave,

(a) $h=0.05$, (b) $h=0.1$, (c) $h=0.2$. 


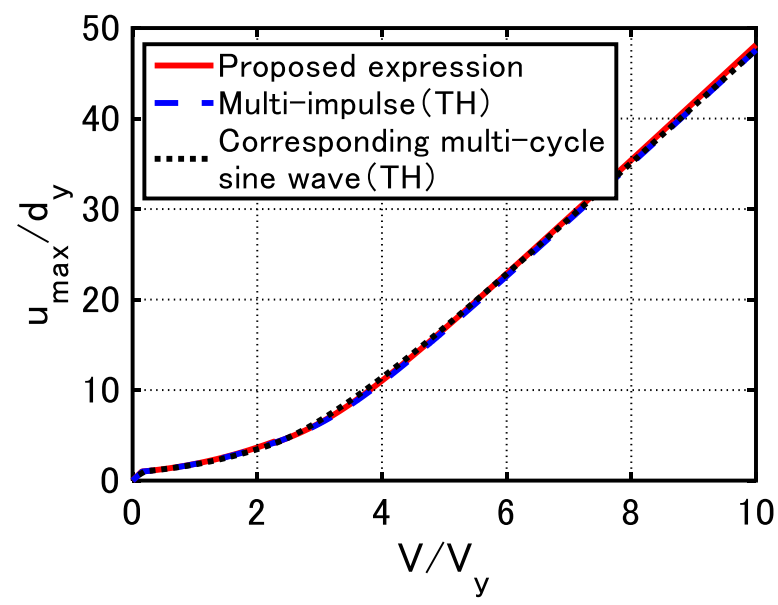

(a)

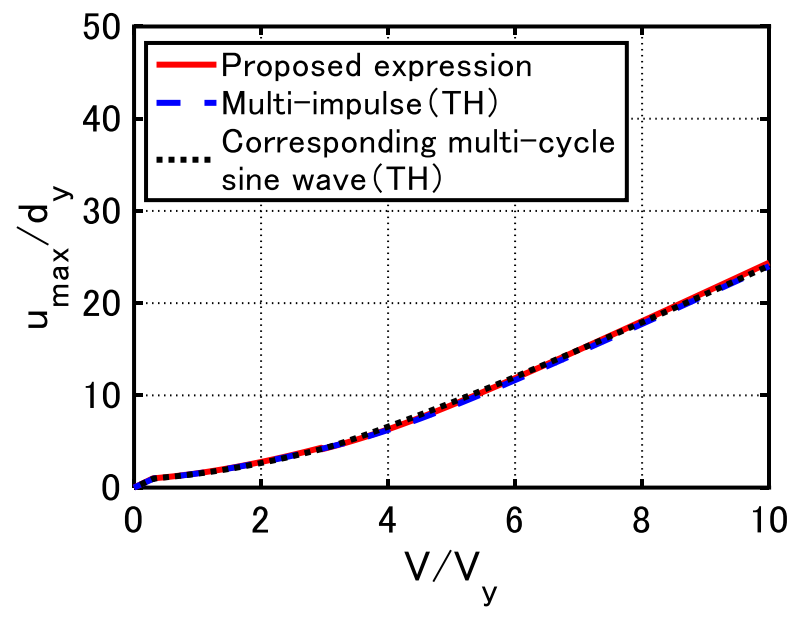

(b)

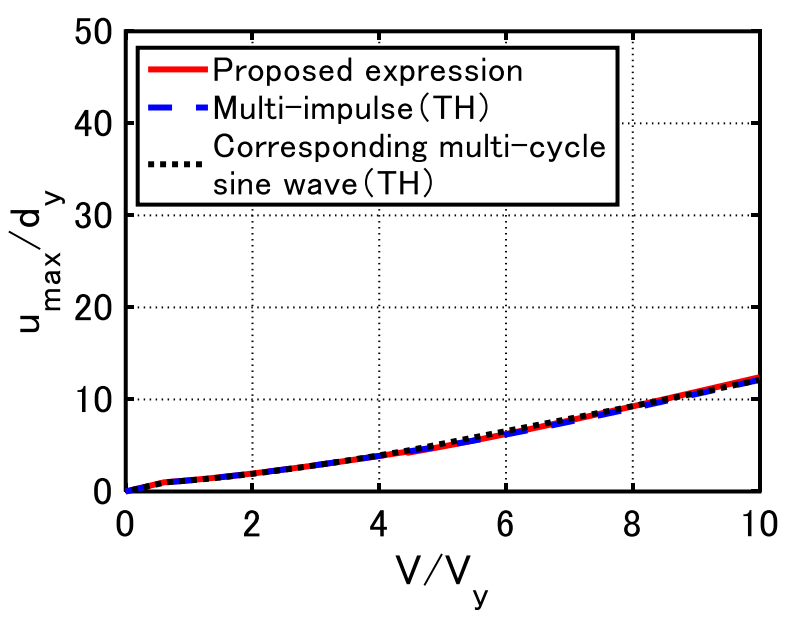

(c)

Figure 8 Comparison of maximum deformations for a sample model of ( $\alpha=0.3$ ), TH response analysis under multi impulse and corresponding multi-cycle sine wave,

(a) $h=0.05$, (b) $h=0.1$, (c) $h=0.2$. 


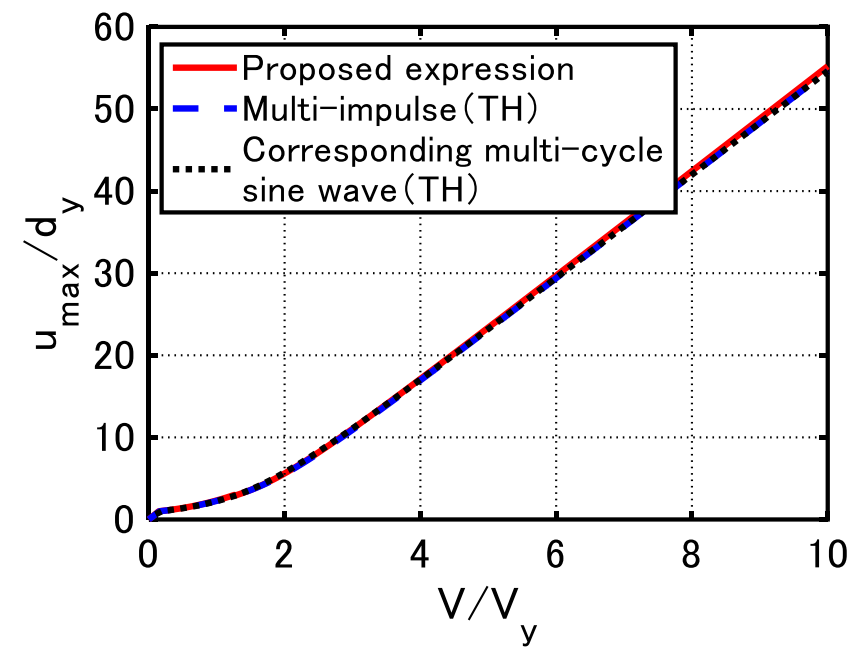

(a)

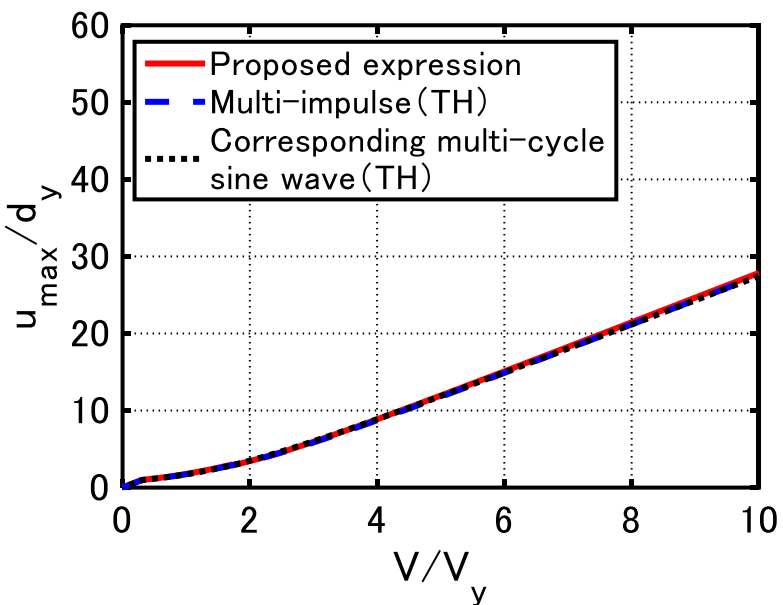

(b)

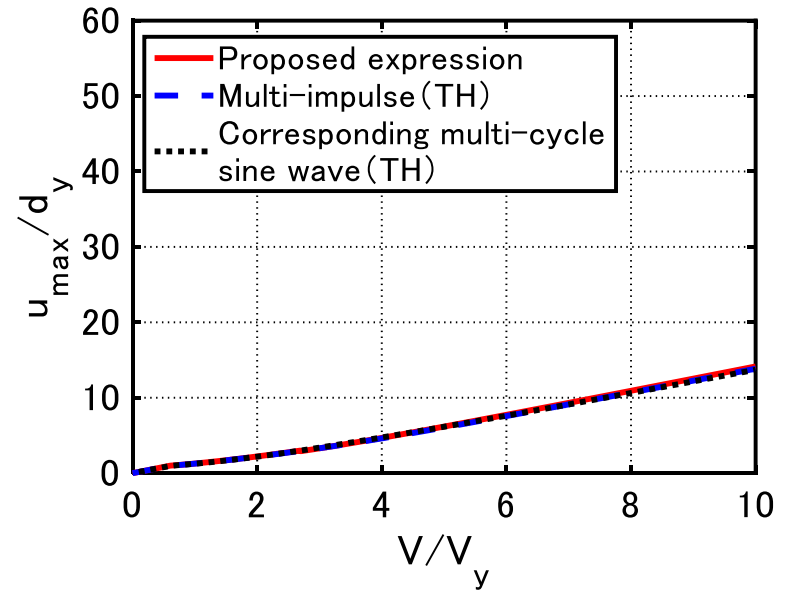

(c)

Figure 9 Comparison of maximum deformations for a sample model ( $\alpha=0.5$ ), TH response analysis under multi impulse and corresponding multi-cycle sine wave,

$$
\text { (a) } h=0.05 \text {, (b) } h=0.1 \text {, (c) } h=0.2 \text {. }
$$

\section{Convergence of impulse timing}

It is investigated In this section, the response for the multi impulse with the constant time interval $t_{0}^{c}$ (Section 3.4) is investigated to see whether it converges to a steady state with each impulse at the zero restoring-force point (see Figure 3). The transient response after the first several cycles of impulses is difficult to achieve, because the number of impulses needed for convergence depends on the level of input velocity and the post-yield stiffness ratio. The TH response analysis is used to determine the response with the constant time interval $t_{0}{ }^{c}$. Consider the parameters $\omega_{1}=1.0(\mathrm{rad} / \mathrm{s}), d_{y}=0.04(\mathrm{~m})$, $\Delta t=1.0 \times 10^{-4} T_{1}$ ( $T_{1}$ : fundamental natural period of the elastic model). $\Delta t$ represents the time step in the $\mathrm{TH}$ response analysis. In case of multi impulse, the response under the multi impulse can be simply calculated by adding $\pm V$ to the mass velocity at the impulse timing. Figures 10 and 11 show the time histories of system responses for the multi impulse with the constant time interval $t_{0}{ }^{c}$ in the model with $\alpha=0.3, h=0.05$ and $V / V_{y}=2.0,6.0$. It is assumed that time interval is determined based on the 
415

416

417

418

419

420

421

422

423

424

425

426

427

428

steady state condition. The acting points of impulses are marked with red circles in Figures 10 and 11. It is shown that the model response converges to a steady state in which each impulse acts at the zero restoring force irrespective to the level of the input velocity. Furthermore, it can be observed that the maximum deformation and the plastic deformation amplitude after convergence coincide with the closed-form formulations in Section 3.2 and Section 3.3. In the model with $\alpha=0.3, h=0.05$ and $V / V_{y}=2.0$ corresponds to CASE 1 in Section 3.2, the impulse acting points converge to the point of zero restoring force in the unloading process (see Figures 10). From Figure 10, the required number of impulses is determined about 15. Figure 11 shows similar results for CASE 2, considering $V / V_{y}=6.0$. From Figure 11, CASE 2 needs about 20 impulses for convergence. Compared with the undamped model (Kojima and Takewaki 2017), a damped bilinear hysteretic model requires a smaller number of impulses for convergence in both of CASE 1 and CASE 2.

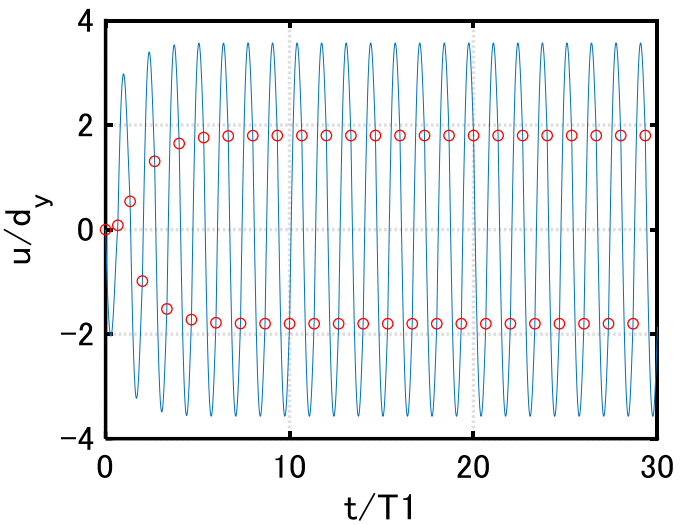

(a)

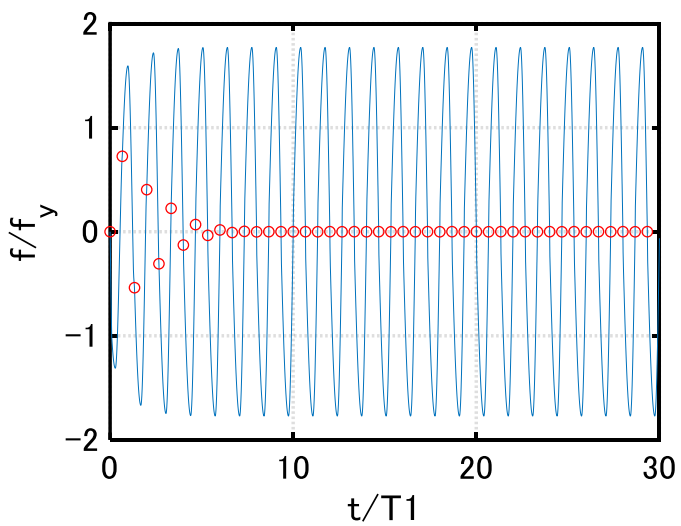

(c)

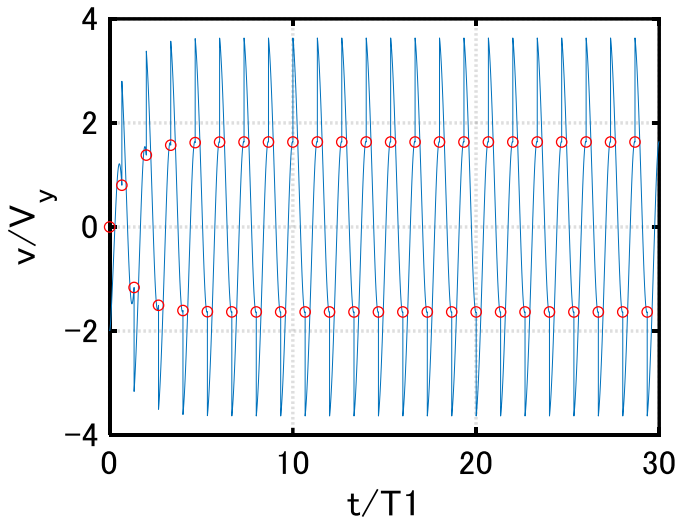

(b)

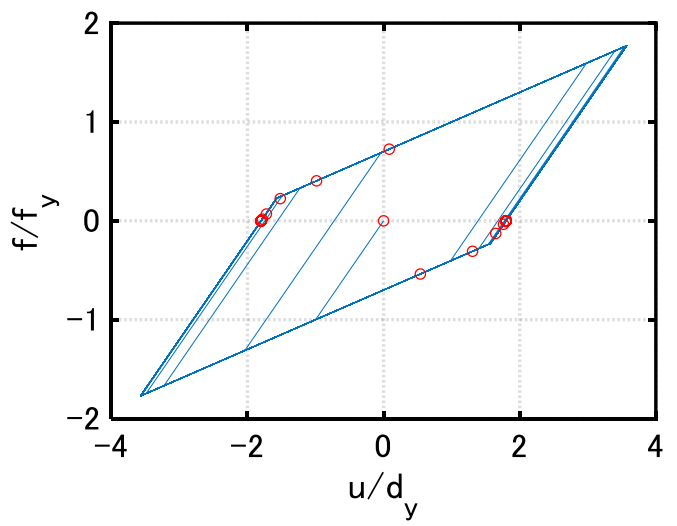

(d)

Figure 10 Model response under multi impulse with time interval $t_{0}{ }^{c}$ for $V / V_{y}=2.0$ and $\alpha=0.3, h=0.05$ (impulse timing is critical one obtained by steady-state assumption):

(a) displacement, (b) velocity, (c) restoring force and (d) restoring force-deformation relation. 
435

436

437

438

439

440

441

442

\section{3}

444

445

446

447

448

449

450

451

452

453

454

455

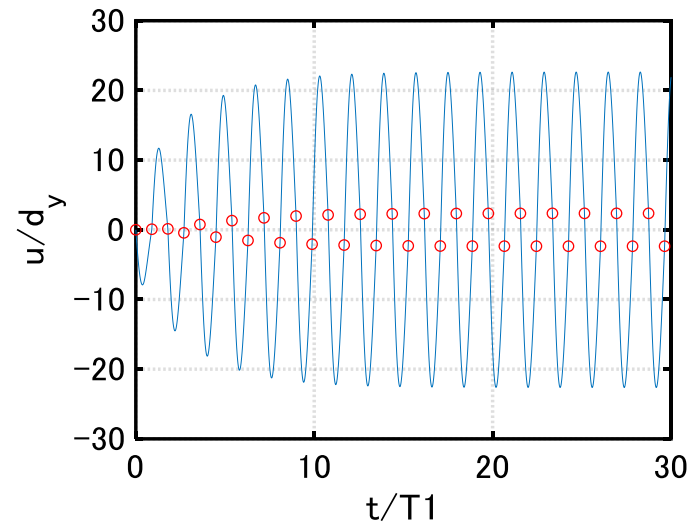

(a)

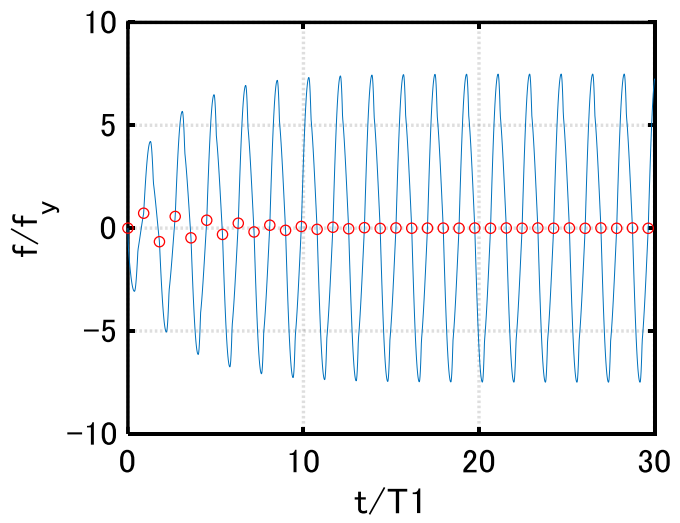

(c)

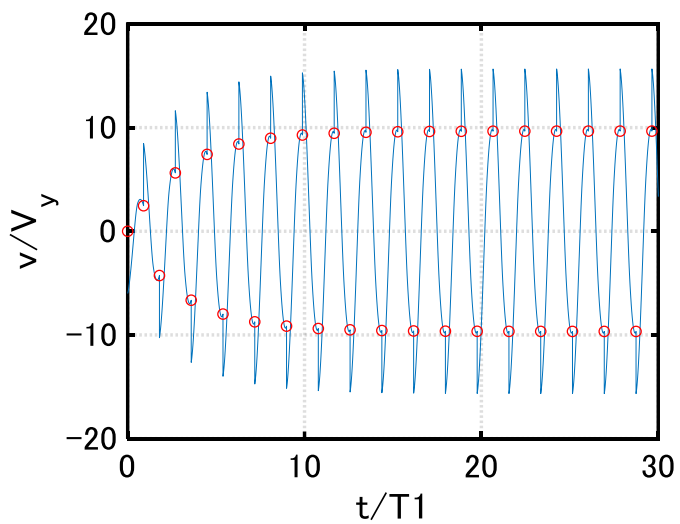

(b)

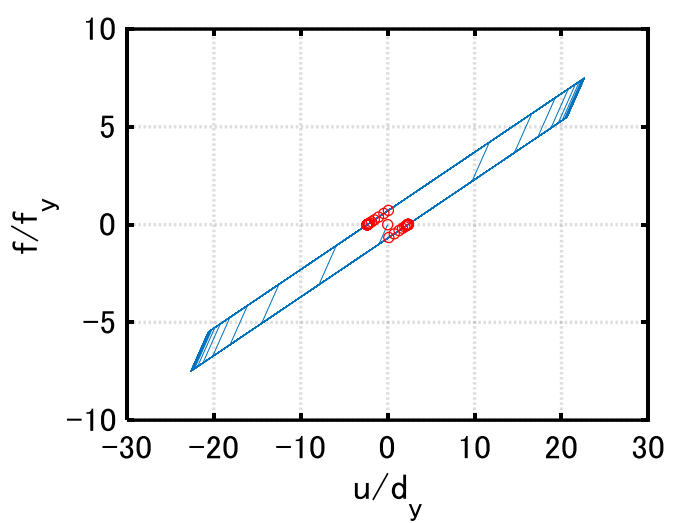

(d)

Figure 11 Model response under multi impulse with time interval $t_{0}^{c}$ for $V / V_{y}=6.0$ and $\alpha=0.3, h=0.05$ (impulse timing is critical one obtained by steady-state assumption):

(a) displacement, (b) velocity, (c) restoring force and (d) restoring force-deformation relation.

\section{Proof of Critical timing proof}

The validity of the critical timing is investigated here. The time-history response analysis has been conducted for the SDOF model subjected to the multi impulse with the various impulse timing $t_{0}$. In the analysis, a variety of input velocity and post-yield stiffness ratios are considered. As discussed in Section 3, it is assumed that the critical timing of each impulse be set as the time with zero restoring force. The parameters $\omega_{1}=1.0(\mathrm{rad} / \mathrm{s}), d_{y}=0.04(\mathrm{~m}), \Delta t=1.0 \times 10^{-4} T_{1}$ are considered in the analysis.

The normalized maximum deformation $u_{\max } / d_{y}$ with respect to the impulse timing $t_{0} / t_{0}{ }^{c}$ normalized by the critical timing for post-yield stiffness ratio $\alpha=0.3$, damping ratio $h=0.05$ and various input velocity levels $V / V_{y}$ are depicted in Figure 12. It is evident that the critical timing $t_{0}{ }^{c}$ derived in Section 3.4 provides the critical one under the multi impulse and gives the upper bound of $u_{\max } / d_{y}$. The closed-form expressions of $u_{\max } / d_{y}$ derived in Sections 3.2 and 3.3 are equal to the upper bound of $u_{\max } / d_{y}$ in Figure 12. 
456

457

458

459

460

461

462

463

464

465

466

467

468

469

470

471

472

473

474

475

476

477

478

479

480

481

482

483

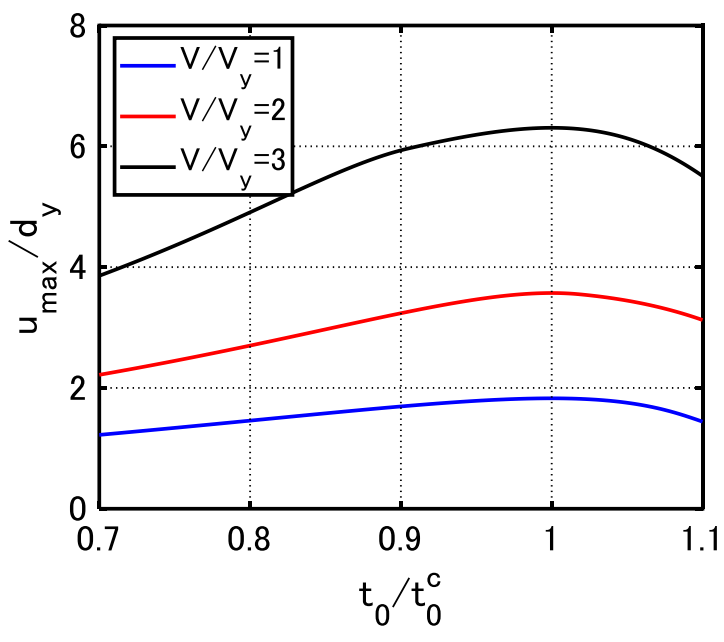

(a)

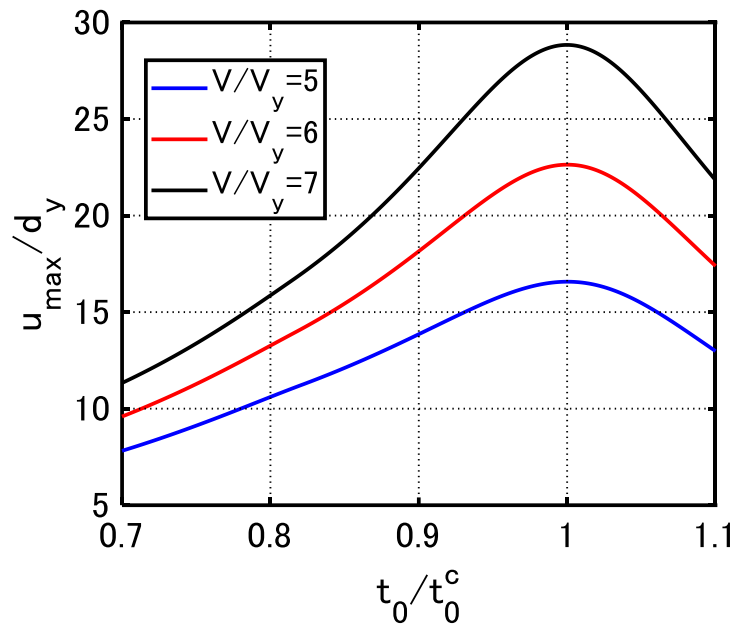

(b)

Figure 12 Maximum deformation with respect to timing of multi impulse for $\alpha=0.3, h=0.05$ and various input levels: (a) $V / V_{y}=1,2,3$, (b) $V / V_{y}=5,6,7$

\section{Applicability of critical multi impulse timing to corresponding sine wave}

In Section 3.5, it was made clear that, if for tuning purpose the maximum Fourier amplitude is selected as the main parameter, a relatively good correspondence will be resulted between the response under the multi impulse with the time interval as shown in Section 3.4 and that under the corresponding multicycle sine wave. In this section, it is investigated whether the critical timing of the multi impulse derived in Section 3.4 can also be a good approximate of the critical period of the multi-cycle sine wave.

In the exact resonance curve (Iwan 1961), the resonant equivalent frequency of the harmonic wave for a specific acceleration amplitude must be obtained. To this aim, it is inevitable to solve the transcendental equation by parametrically changing the frequency of excitation.

To compute the response of SDOF model for the multi-cycle sine wave with various period $\left(T_{l}\right)$, various levels of input velocity and various post-yield stiffness ratios, the TH response analysis has been conducted subjected to multi-cycle sine wave. $T_{l}, \omega_{l}=2 \pi / T_{l}, A_{l}$ and $V_{l}=A_{l} / \omega_{l}$ are as described in Section 3.5, for the case of sine wave tuned to the multi impulse with the constant time interval $t_{0}$ and the input velocity level $V$. It is assumed that the input period $T_{l}=2 t_{0}$. It should be noted that the input period should be changed for the specific velocity amplitude calculated by Eq. (33) with the input velocity level $V$. The critical period of the multi-cycle sine wave is characterized as $T_{l}^{c}=2 t_{0}^{c}$ for a specific velocity amplitude $V_{l}$.

The model responses $\left(u_{\max } / d_{y}\right.$ and $\left.u_{p} / d_{y}\right)$ with respect to the input period $T_{l} / T_{l}^{c}\left(=t_{0} / t_{0}{ }^{c}\right)$ normalized by the approximate critical period for $\alpha=0.3, h=0.05$ and various input velocity levels $V / V_{y}$ are illustrated in Figure 13. It can be seen that $T_{l}^{c}=2 t_{0}{ }^{c}$ is a fairly good approximate of the critical period of the multi-cycle sine wave for a specific velocity amplitude. 


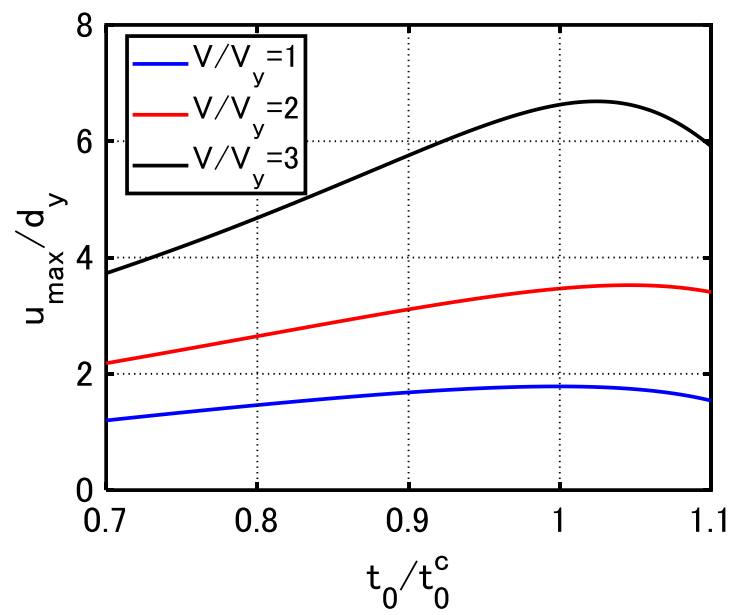

(a)

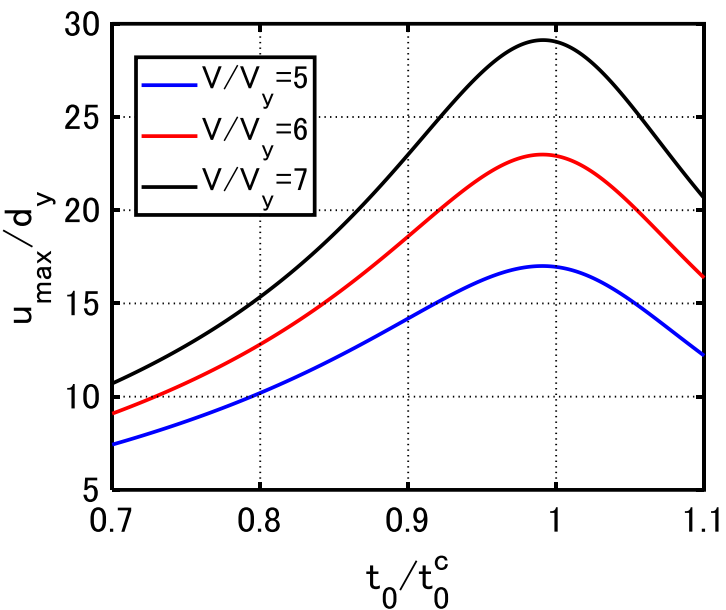

(b)

Figure 13 Maximum deformation with respect to period of corresponding sine wave for $\alpha=0.3, h=0.05$ and various input levels: (a) $V / V_{y}=1,2,3$, (b) $V / V_{y}=5,6,7$

\section{Exact solution and verification}

The comparison with the resonance curve under the sine wave computed by using the exact solution (Iwan 1961) is made for the accuracy check of the proposed expression on the steady-state response under the critical multi impulse. In the conventional curve given by (Iwan 1961), the transcendental equation has to be solved by parametrically changing the excitation frequency and the resonant equivalent frequency of the harmonic wave for a specific acceleration amplitude should be extracted from the given curve. On the other hand, the proposed method gives a closed form solution for the direct critical steady-state response under a specific input level. The input level of the multi impulse and the corresponding sine wave has been tuned by using the equivalence of the maximum Fourier amplitude as described in previous sections.

A comparison between the proposed formulation and the conventional resonance curve proposed by Iwan (1961) is made in Figure 14. The parameter $\omega^{*}$ in Figure 14 denotes the ratio of the excitation frequency $\omega_{l}=2 \pi / T_{l}$ of the corresponding sine wave to the elastic natural circular frequency $\omega_{1}$. In addition, $r$ indicates the ratio of the excitation acceleration amplitude $A_{l}=\omega_{l} V_{l}$ of the corresponding sine wave to the parameter $A_{y}=\omega_{1}^{2} d_{y} . r=A_{l} / A_{y}$ is also equal to the product of the normalized acceleration amplitude by $f_{y}$ and the model mass. The red line in Figure 14 indicates the maximum deformation under the critical multi impulse. The red solid circles in the red line present the input levels of the multi impulse. Considering $T_{l}=2 t_{0}{ }^{c}$ in the critical case, the normalized critical timing $t_{0}{ }^{c} / T_{1}$ can be converted to $\omega^{*}=T_{1} /\left(2 t_{0}{ }^{c}\right)$. The critical time interval obtained in Section 3.4 is used for $t_{0}{ }^{c}$. The black line in Figures 14(a) and 14(b) are representing the resonance curves with $r=0.552,0.955,1.23$ and $r=1.80,2.13,2.46$ respectively.. In addition, the blue dotted lines in Figure 14 presents the resonance curve for constant velocity amplitude. From Figure 14(a), it is evident that the proposed closed-form expression corresponds much better to the blue dotted lines than the black lines for the case of small input levels. With the large input levels, the resonance point in the blue 
513

514

515

516

517

518

519

520

521

522

523

524

525

526

527

528

529

530

531

532

533

534

535

536

537

538

539

540

dotted line and that in the black line exhibit a fairly good correspondence. Therefore, the proposed formulation on the critical maximum deformation under the multi impulse corresponds well to both.

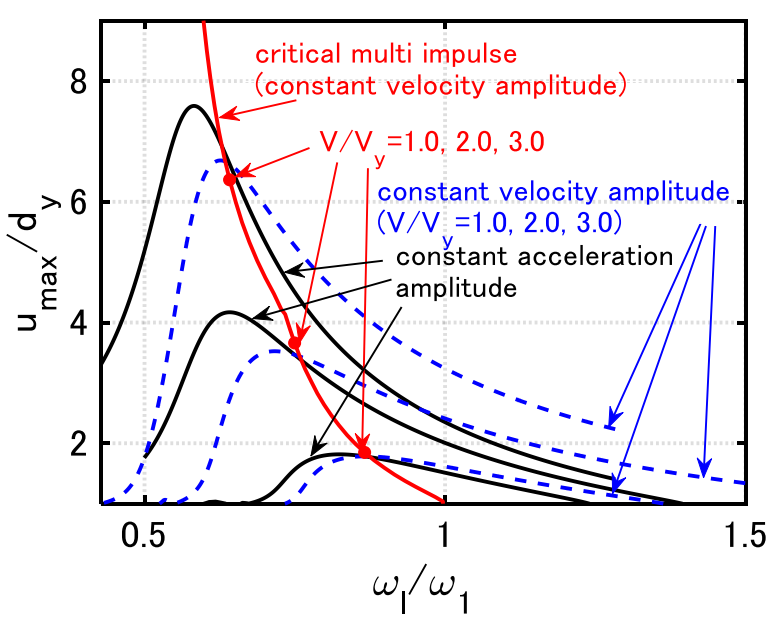

(a)

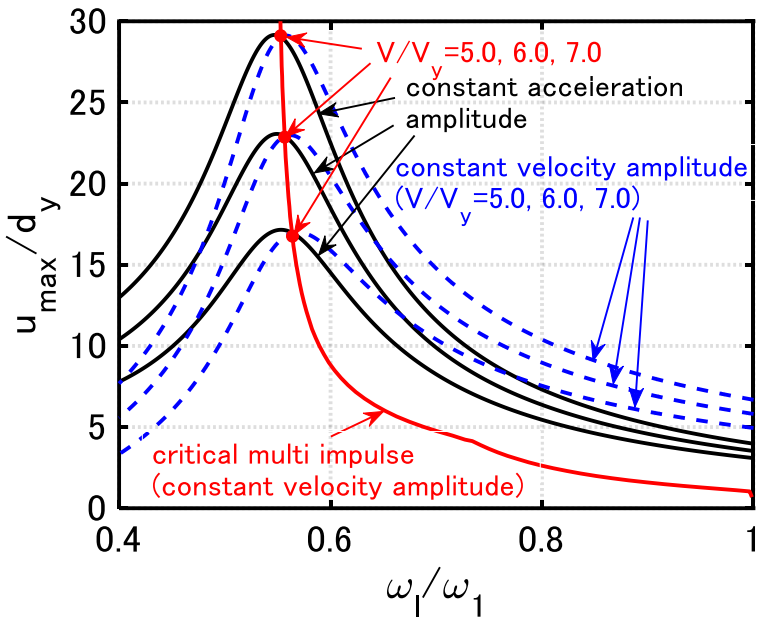

(b)

Figure 14 Comparison of closed-form maximum deformation under critical multi impulse resonance curves under sine wave for $\alpha=0.3, h=0.05$ and various input levels:

(a) $V / V_{y}=1,2,3$, (b) $V / V_{y}=5,6,7$

\section{Conclusions}

The long-duration GM has been modeled by the multi impulse and the closed-form solution has been derived for the maximum steady-state response of a damped bilinear hysteretic SDOF model subjected to the critical multi impulse. While the conventional method for the sine wave (Iwan 1961) requires the resonant equivalent frequency which can be computed by changing the excitation frequency parametrically, the steady-state response under the critical multi impulse can be obtained in closedform (without repetition) for the variating input level in the proposed approach. The conclusions are as follows:

(1) It is assumed that the system is in he steady state in which each impulse acts at the point of zero restoring-force, and the closed-form critical response have been derived implementing an energybased approach under the critical multi impulse have been derived by using the energy balance law and the approximation (quadratic or elliptical) of the damping force-deformation relation of the dashpot. Considering the level of input velocity and the plastic deformation, the critical steady state is classified into three cases. In CASE 0, the model remains elastic. In CASEs 1 and 2, each impulse acts at the point of zero restoring-force in the unloading and loading processes respectively.

(2) In case of constant time interval, the model response will be converged to steady state as depicted in Figure 3. The model responses after convergence into the steady state correspond to the closedform expressions. 
541

(3) The reliability and accuracy of the derived closed-form solution have been verified through the TH response analysis. The comparison has been made between the steady-state response under the critical multi impulse and that under the corresponding multi-cycle sinusoidal wave as a representative of the long-duration GM. The results clearly confirmed the multi impulse is an acceptable substitute of the multi-cycle sinusoidal wave in the evaluation model response if the maximum Fourier amplitude is tuned. Compared with the undamped model, the responses of a damped bilinear hysteretic SDOF under those inputs correspond better.

(4) The critical time interval can be derived using the TH response analysis in Section 3.4. The validity of the critical time interval is confirmed by the analysis for the damped bilinear hysteretic SDOF model subjected to the multi impulse under a variety of impulse timing. The critical timing of each impulse is defined as the time with zero restoring-force in the steady state.

(5) Double of the critical time interval is a good approximate of the critical period of the multi-cycle sinusoidal wave with the corresponding input amplitude.

In the previous paper (Akehashi et al., 2018a), a closed-form critical response was derived for a damped bilinear hysteretic SDOF model subjected to the double impulse. That proposed methodology was extended to the problem of critical excitation for a base-isolated building structure on ground under a near-fault GM (double impulse) by reducing the structural model into an SDOF system (Akehashi et al., 2018b). The approach proposed in this paper can also be extended to the problem of critical excitation for a base-isolated structure under long-duration motion.

\section{Acknowledgement}

Part of the present work is supported by the Grant-in-Aid for Scientific Research (KAKENHI) of Japan Society for the Promotion of Science (No.17K18922, 18H01584) and Sumitomo Rubber Industries, Co. This support is greatly appreciated.

\section{References}

Abrahamson, N., Ashford, S., Elgamal, A., Kramer, S., Seible, F., and Somerville, P. (1998). 1st PEER Workshop on Characterization of Special Source Effects, Pacific Earthquake Engineering Research Center, University of California, San Diego, 1998.

Akehashi, H., Kojima, K., and Takewaki, I. (2018a). Critical response of SDOF damped bilinear hysteretic system under double impulse as substitute for near-fault ground motion. Frontiers in Built Environment, 4: 5.

Akehashi H., Kojima K., Fujita K., and Takewaki, I. (2018b). Critical Response of Nonlinear BaseIsolated Building Considering Soil-Structure Interaction Under Double Impulse as Substitute for Near-Fault Ground Motion. Frontiers in Built Environment, 4: 34. 
577

578

579

580

581

582

583

584

585

586

587

588

589

590

591

592

593

594

595

596

597

598

599

600

601

602

603

604

605

606

607

608

609

610

611

612

613

614

Bertero, V. V., Mahin, S. A., and Herrera, R. A. (1978). Aseismic design implications of near-fault San Fernando earthquake records, Earthquake Eng. Struct. Dyn. 6(1), 31-42.

Casapulla, C., Jossa, P. and Maione, A. (2010). Rocking motion of a masonry rigid block under seismic actions: a new strategy based on the progressive correction of the resonance response. Ingegneria Sismica; 27(4): 35-48.

Casapulla, C. and Maione, A. (2016). Free damped vibrations of rocking rigid blocks as uniformly accelerated motions. Int. J. of Structural Stability and Dynamics, http://dx.doi.org/10.1142/ S0219455417500584.

Caughey, T. K. (1960a). Sinusoidal excitation of a system with bilinear hysteresis. J. Appl. Mech. 27, 640-643. doi:10.1115/1.3644077

Caughey, T. K. (1960b). Random excitation of a system with bilinear hysteresis. J. Appl. Mech. 27, 649-652. doi:10.1115/1.3644077

Iwan, W. D. (1961). The dynamic response of bilinear hysteretic systems, Ph.D. Thesis, California Institute of Technology, Pasadena.

Iwan, W. D. (1965a). The dynamic response of the one-degree-of-freedom bilinear hysteretic system, Proc. of the Third World Conf. on Earthquake Eng., New Zealand.

Iwan, W. D. (1965b). The steady-state response of a two-degree-of-freedom bilinear hysteretic system, J. Applied Mech., 32(1), 151-156.

Kalkan, E., and Kunnath, S.K. (2006). Effects of fling step and forward directivity on seismic response of buildings, Earthquake Spectra, 22(2), 367-390.

Kojima, K., and Takewaki, I. (2015a). Critical earthquake response of elastic-plastic structures under near-fault ground motions (Part 1: Fling-step input), Frontiers in Built Environment, 1: 12.

Kojima, K., and Takewaki, I. (2015b). Critical earthquake response of elastic-plastic structures under near-fault ground motions (Part 2: Forward-directivity input), Frontiers in Built Environment, 1: 13.

Kojima, K., and Takewaki, I. (2015c). Critical input and response of elastic-plastic structures under long-duration earthquake ground motions, Frontiers in Built Environment, 1: 15.

Kojima, K., and Takewaki, I. (2016). Closed-form critical earthquake response of elastic-plastic structures with bilinear hysteresis under near-fault ground motions, J. Struct. Construction Eng., AIJ, 726, 1209-1219 (in Japanese).

Kojima, K., and Takewaki, I. (2017). Critical steady-state response of single-degree-of-freedom bilinear hysteretic system under multi impulse as substitute of long-duration ground motion, Frontiers in Built Environment, 3: 41.

Kojima, K., Saotome, Y., and Takewaki, I. (2017). Critical earthquake response of a SDOF elasticperfectly plastic model with viscous damping under double impulse as a substitute of near-fault ground motion, J. Struct. Construction Eng., AIJ, 735, 643-652 (in Japanese), https://doi.org/10.3130/aijs.82.643. [English Version] Japan Architectural Review, Wiley, 2018, doi: 10.1002/2475-8876.10019. 
615 Liu, C.-S. (2000). The steady loops of SDOF perfectly elastoplastic structures under sinusoidal 616 loadings. J. Mar. Sci. Technol. 8, 50-60.

617 Roberts, J. B., and Spanos, P. D. (1990). Random Vibration and Statistical Linearization. New York: 618 Wiley.

619 Takewaki, I., Murakami, S., Fujita, K., Yoshitomi, S., and Tsuji, M. (2011). The 2011 off the Pacific coast of Tohoku earthquake and response of high-rise buildings under long-period ground motions, Soil Dyn. Earthquake Eng., 31(11), 1511-1528. 